\title{
Time-phase bispectral analysis
}

\author{
Janez Jamšek, ${ }^{1,2,3, *}$ Aneta Stefanovska, ${ }^{1,3, \dagger}$ Peter V. E. McClintock, ${ }^{3, \sharp}$ and Igor A. Khovanov ${ }^{3,4, \S}$ \\ ${ }^{1}$ Group of Nonlinear Dynamics and Synergetics, Faculty of Electrical Engineering, University of Ljubljana, Tržaška 25, \\ 1000 Ljubljana, Slovenia \\ ${ }^{2}$ Department of Physics and Technical Studies, Faculty of Education, University of Ljubljana, Kardeljeva ploščad 16, \\ 1000 Ljubljana, Slovenia \\ ${ }^{3}$ Department of Physics, University of Lancaster, Lancaster LA1 4YB, United Kingdom \\ ${ }^{4}$ Laboratory of Nonlinear Dynamics, Saratov State Unversity, 410026 Saratov, Russia
}

(Received 8 January 2003; published 3 July 2003)

\begin{abstract}
Bispectral analysis, a technique based on high-order statistics, is extended to encompass time dependence for the case of coupled nonlinear oscillators. It is applicable to univariate as well as to multivariate data obtained, respectively, from one or more of the oscillators. It is demonstrated for a generic model of interacting systems whose basic units are the Poincaré oscillators. Their frequency and phase relationships are explored for different coupling strengths, both with and without Gaussian noise. The distinctions between additive linear or quadratic, and parametric (frequency modulated), interactions in the presence of noise are illustrated.
\end{abstract}

DOI: 10.1103/PhysRevE.68.016201

PACS number(s): 05.45.Df, 02.70.Hm, 05.45.Xt

\section{INTRODUCTION}

Most real systems are nonlinear and complex. In general, they may be regarded as a set of interacting subsystems; given their nonlinearity, the interactions can be expected to be nonlinear too.

The phase relationships between a pair of interacting oscillators can be obtained from bivariate data (i.e., where the coordinate of each oscillator can be measured separately) by use of the methods recently developed for analysis of synchronization, or generalized synchronization, between chaotic and/or noisy systems. Not only can the interactions be detected [1], but their strength and direction can also be determined [2]. The next logical step in studying the interactions among coupled oscillators must be to determine the nature of the couplings: the methods developed for synchronization analysis are not capable of answering this question.

Studies of higher-order spectra, or polyspectra, offer a promising way forward. The approach is applicable to interacting systems quite generally, regardless of whether or not they are mutually synchronized. Following the pioneering work of Brillinger and Rosenblatt [3], increasing applications of polyspectra in a diversity of fields have appeared, e.g., telecommunications, radar, sonar, speech, biomedical, geophysics, imaging systems, surface gravity waves, acoustics, econometrics, seismology, nondestructive testing, oceanography, plasma physics, and seismology. An extensive overview can be found in Ref. [4]. The use of the bispectrum as a means of investigating the presence of second-order nonlinearity in interacting harmonic oscillators has been of particular interest during the last few years [5-8].

Systems are usually taken to be stationary. For real systems, however, the mutual interaction among subsystems of-

\footnotetext{
*Electronic address: janez.jamsek@pef.uni-lj.si

${ }^{\dagger}$ Electronic address: aneta@osc.fe.uni-lj.si

‡Electronic address: p.v.e.mcclintock@lancaster.ac.uk

${ }^{\S}$ Electronic address: igor@chaos.ssu.runnet.ru
}

ten results in time variability of their characteristic frequencies. Frequency and phase couplings can occur temporally, and the strength of coupling between pairs of individual oscillators may change with time. In studying such systems, bispectral analysis for stationary signals, based on time averages, is no longer sufficient. Rather, the time evolution of the bispectral estimates is needed.

Priestley and Gabr [9] were probably the first to introduce the time-dependent bispectrum for harmonic oscillators. Most of the subsequent work has been related to the timefrequency representation and is based on high-order cumulants [10]. The parametric approach has been used to obtain approximate expressions for the evolutionary bispectrum [11]. Further, Perry and Amin have proposed a recursion method for estimating the time-dependent bispectrum [12]. Dandawaté and Giannakis have defined estimators for cyclic and time-varying moments and cumulants of cyclostationary signals [13]. Schack et al. [14] have recently introduced a time-varying spectral method for estimating the bispectrum and bicoherence: the estimates are obtained by filtering in the frequency domain and then obtaining a complex timefrequency signal by inverse Fourier transform. They assume, however, that the interacting oscillators are harmonic.

Millingen et al. [15] introduced the wavelet bicoherence and were the first to demonstrate the use of bispectra for studying interactions among nonlinear oscillators. They used the method to detect periodic and chaotic interactions between two coupled van der Pol oscillators, but without concentrating on time-phase relationships, in particular.

In this paper we develop an approach [16] that introduces time dependance to the bispectral analysis of univariate data. We focus on the time-phase relationships between two (or more) interacting systems. As we demonstrate below, the method enables us to detect that two or more subsystems are interacting with each other, to quantify the strength of the interaction, and to determine its nature, whether additive linear or quadratic, or parametric in one of the frequencies. It yields results that are applicable quite generally to any system of coupled nonlinear oscillators. Our principal motiva- 
tion has been to develop a technique for studying the human cardiovascular system [17], including the interactions among its subsystems, and the nature of these interactions. Here, however, we are concerned with basic principles, and in demonstrating (testing) the technique on a well-characterized simple model. Application to the more challenging problem posed by the cardiovascular system, currently in progress, will be described in a subsequent publication.

\section{METHOD}

\section{A. Bispectral analysis}

Bispectral analysis belongs to a group of techniques based on high-order statistics (HOS) that may be used to analyze non-Gaussian signals, to obtain phase information, to suppress Gaussian noise of unknown spectral form, and to detect and characterize signal nonlinearities [5]. In what follows we extend bispectral analysis to extract useful features from nonstationary data, and we demonstrate the modified technique by application to test signals generated from coupled oscillators.

The bispectrum involves third-order statistics. Spectral estimation is based on the conventional Fourier type direct approach, through computation of the third-order moments which, in the case of third-order statistics, are equivalent to third-order cumulants $[5,18-21]$.

The classical bispectrum estimate is obtained as an average of estimated third-order moments (cumulants) $\hat{M}_{3}^{i}(k, l)$,

$$
\hat{B}(k, l)=\frac{1}{K} \sum_{i=1}^{K} \hat{M}_{3}^{i}(k, l),
$$

where the third-order moment estimate $\hat{M}_{3}^{i}(k, l)$ is performed by a triple product of discrete Fourier transforms (DFTs) at discrete frequencies $k, l$, and $k+l$ :

$$
\hat{M}_{3}^{i}(k, l)=X_{i}(k) X_{i}(l) X_{i}^{*}(k+l),
$$

with $i=1, \ldots, K$ segments into which the signal is divided to try to obtain statistical stability of the estimates, see the Appendix.

Just as the discrete power spectrum has a point of symmetry at the folding frequency $f_{s} / 2$, the discrete bispectrum has many symmetries in the $(k, l)$ plane [22]. Because of these, it is necessary to calculate the bispectrum only in the nonredundant region, or principal domain, as shown in Fig. 1. The principal domain can be divided into two triangular regions in which the discrete bispectrum has different properties: the inner triangle (IT) and the outer one [23]. In the current work it is the IT that is of primary interest. Thus, it is sufficient to calculate the bispectrum over the IT of the principal domain defined in Refs. [5,7]: $0 \leqslant l \leqslant k, k+l \leqslant f_{s} / 2$.

The bispectrum $B(k, l)$ is a complex quantity, defined by magnitude $A$ and phase $\phi$,

$$
B(k, l)=|B(k, l)| e^{j \angle B(k, l)}=A e^{j \phi} .
$$

Consequently, for each $(k, l)$, its value can be represented as a point in a complex space, $\operatorname{Re}[B(k, l)]$ versus $\operatorname{Im}[B(k, l)]$,

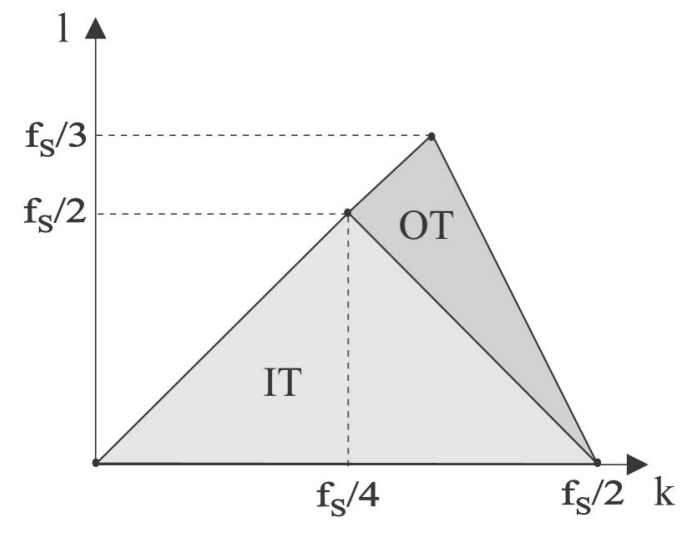

FIG. 1. The principal domain of the discrete bispectrum of a band-limited signal can be divided into two triangular regions, the inner triangle (IT) and the outer triangle (OT). $k$ and $l$ are discrete frequencies, $f_{S}$ is the sampling frequency.

thus defining a vector. Its magnitude (length) is known as the biamplitude. The phase, which for the bispectrum is called the biphase, is determined by the angle between the vector and the positive real axis.

The bispectrum quantifies the relationships among the underlying oscillatory components of the observed signals. Specifically, bispectral analysis examines the relationships between the oscillations at two basic frequencies, $k$ and $l$, and a harmonic component at the frequency $k+l$. This set of three frequencies is known as a triplet $(k, l, k+l)$. The bispectrum $B(k, l)$, a quantity incorporating both phase and power information, can be calculated for each triplet.

A high bispectrum value at bifrequency $(k, l)$ indicates that there is at least frequency coupling within the triplet of frequencies $k, l$, and $k \pm l$. Strong coupling implies that the oscillatory components at $k$ and $l$ may have a common generator. Such components may synthesize a new component at the combinatorial frequency $k \pm l$ if a quadratic nonlinearity is present.

\section{B. Time-phase bispectral analysis}

The classical bispectral method is adequate for studying stationary signals whose frequency content is preserved over time. We now wish to encompass time dependance within the bispectral analysis. In analogy with the short-time Fourier transform, we accomplish this by moving a time window $w(n)$ of length $M$ across the signal $x(n)$, calculating the DFT at each window position

$$
X(k, n) \cong \frac{1}{M} \sum_{n=0}^{M-1} x(n) w(n-\tau) e^{-j 2 \pi n k / M} .
$$

Here, $k$ is the discrete frequency, $n$ the discrete time, and $\tau$ the time shift. The choice of window length $M$ is a compromise between achieving optimal frequency resolution and optimal detection of the time variability. The instantaneous biphase is then calculated: from Eqs. (2) and (3), it is

$$
\phi(k, l, n)=\phi_{k}(n)+\phi_{l}(n)-\phi_{k+l}(n) .
$$



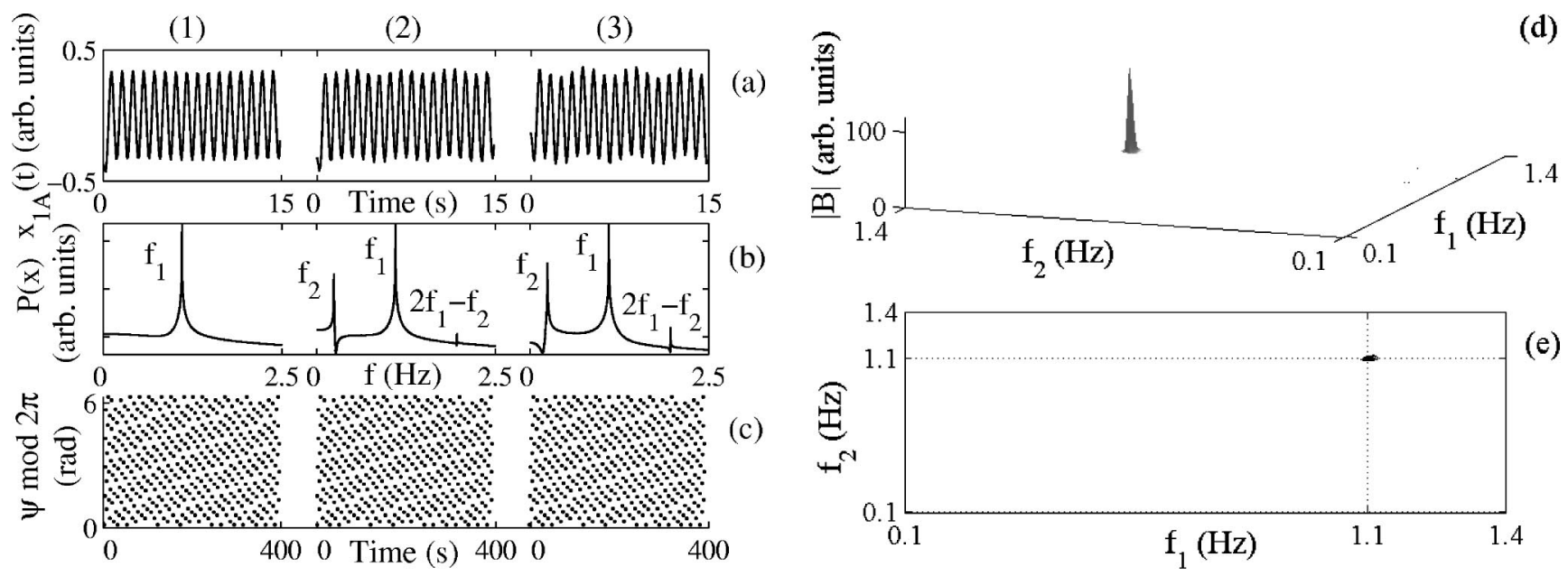

FIG. 2. Results in the absence of noise. (a) The test signal $x_{1 A}(t)$, variable $x_{1}$ of the first oscillator with characteristic frequency $f_{1}$ $=1.1 \mathrm{~Hz}$. The characteristic frequency of the second oscillator is $f_{2}=0.24 \mathrm{~Hz}$. The oscillators are unidirectionally and linearly coupled with three different coupling strengths: $\eta_{2}=0.0(1), 0.1(2)$, and $0.2(3)$. Each coupling lasts for $400 \mathrm{~s}$ at sampling frequency $f_{s}=10 \mathrm{~Hz}$. Only the first $15 \mathrm{~s}$ are shown in each case. (b) The power spectrum and (c) synchrogram. (d) The bispectrum $|B|$, using $K=33$ segments, 66\% overlapping, and the Blackman window to reduce leakage and (e) its contour view.

If the two frequency components $k$ and $l$ are frequency and phase coupled, $\phi_{k+l}=\phi_{k}+\phi_{l}$, it holds that the biphase is 0 $(2 \pi)$ radians. For our purposes the phase coupling is less strict because dependent frequency components can be phase delayed. We consider phase coupling to exist if the biphase is constant (but not necessarily $=0$ radians) for at least several periods of the lowest frequency component. Simultaneously, we observe the instantaneous biamplitude from which it is possible to infer the relative strength of the interaction. We thus hope to be able to observe the presence and persistence of coupling among the oscillators.

\section{ANALYSIS}

To illustrate the essence of the method, and to test it, we use a generic model of interacting systems whose basic unit is the Poincare oscillator:

$$
\begin{aligned}
& \dot{x}_{i}=-x_{i} q_{i}-\omega_{i} y_{i}+g_{x_{i}}, \\
& \dot{y}_{i}=-y_{i} q_{i}+\omega_{i} x_{i}+g_{y_{i}}, \\
& q_{i}=\alpha_{i}\left(\sqrt{x_{i}^{2}+y_{i}^{2}}-a_{i}\right) .
\end{aligned}
$$

Here $x$ and $y$ are vectors of the oscillator state variables, $\alpha_{i}$, $a_{i}$ and $\omega_{i}$ are constants, and $g_{y}(y)$ and $g_{x}(x)$ are coupling vectors. The activity of each subsystem is described by the two state variables $x_{i}$ and $y_{i}$, where $i=1, \ldots, N$ denotes the subsystem.

The form of the coupling terms can be adjusted to study different kinds of interaction among the subsystems, e.g., additive linear or quadratic, or parametric frequency modulation. Examples will be considered both without and with a zero-mean white Gaussian noise to obtain more realistic conditions.
Different cases of interaction are demonstrated for signals generated by the proposed model. In each case we analyze the $x_{1}$ variable of the first oscillator, recorded as a continuous time series. For the first $400 \mathrm{~s}$, the interoscillator coupling strength was zero. It was then raised to a small constant value. After a further $400 \mathrm{~s}$, it was increased again. The first $15 \mathrm{~s}$ and corresponding power spectrum for each coupling strength are shown in the figures for each test signal, in order to demonstrate the changes in spectral content and behavior caused by the coupling. For bispectral analysis the whole signal is analyzed as a single entity, but the transients caused by the changes in coupling strength are removed prior to processing. First the classical bispectrum is estimated. Bifrequencies where peaks provide evidence of possible frequency interactions are then further studied by the calculation of the biphase and biamplitude as functions of time. They were calculated using a window of length $100 \mathrm{~s}$, moved across the signal in $0.3 \mathrm{~s}$ steps.

\section{A. Linear couplings}

Let us start with the simplest case of a linear interaction between coupled oscillators. We suppose model (6) to consist of only two oscillators, $i=1,2$. The parameters of the model are set to $\alpha_{1}=1, a_{1}=0.5$ and $\alpha_{2}, a_{2}=1$. The coupling term is unidirectional and linear

$$
g_{x_{1}}=\eta_{2} x_{2}, \quad g_{y_{1}}=\eta_{2} y_{2} .
$$

The test signal $x_{1 A}(t)$ is the variable $x_{1}$ of the first oscillator. It is presented in Fig. 2(a) with the corresponding power spectrum for three different coupling strengths: no coupling $\eta_{2}=0$ and weak couplings $\eta_{2}=0.1,0.2$. The peaks labeled as $f_{1}=1.1 \mathrm{~Hz}$ and $f_{2}=0.24 \mathrm{~Hz}$ are the independent harmonic components of the first and the second oscillator. These frequencies are deliberately chosen to approximately have a noninteger ratio. There is also at least one peak 

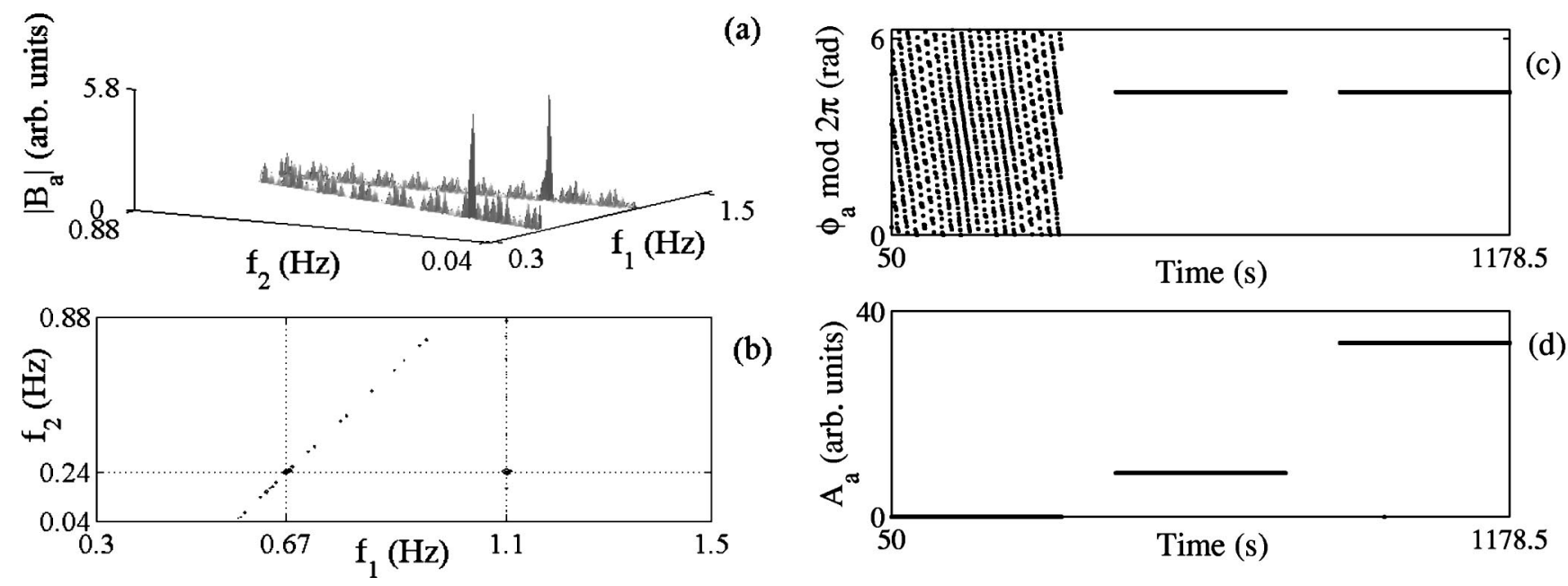

FIG. 3. (a) Adapted bispectrum $\left|B_{a}\right|$, calculated from the test signal $x_{1 A}$ using $K=34$ segments, $80 \%$ overlapping, and the Blackman window and (b) its contour view. Regions of the adapted bispectrum above $f_{2}>0.88 \mathrm{~Hz}$ and below $f_{1}<0.3 \mathrm{~Hz}$ are cut, because the triplets $(1.1 \mathrm{~Hz}, 1.1 \mathrm{~Hz}, 1.1 \mathrm{~Hz})$ and $(0.24 \mathrm{~Hz}, 0.24 \mathrm{~Hz}, 0.24 \mathrm{~Hz})$ produce high peaks that are physically meaningless. (c) Adapted biphase $\phi_{a}$ and (d) biamplitude $A_{a}$ for bifrequency $(1.1 \mathrm{~Hz}, 0.24 \mathrm{~Hz})$, using a 0.3 -s time step and a 100-s-long Blackman window for estimating the DFT.

present at the harmonically related position $f_{3}=2 f_{1}-f_{2}$ attributable to interaction between the two oscillators. It arises from the nonlinearity of the first oscillator, but is caused by the forcing of the second oscillator.

The principal domain of the bispectrum for the test signal $x_{1 A}$, Fig. 2(d), shows one peak at the bifrequency $(1.1 \mathrm{~Hz}$, $1.1 \mathrm{~Hz})$, the so-called self-coupling. No other peaks are present. Bispectral analysis examines the relationships between oscillations at the two basic frequencies $f_{1}$ and $f_{2}$, and a modulation component at the frequency $f_{1} \pm f_{2}$, which is absent from the power spectra in Fig. 2(b). Therefore, no peak is present at bifrequency $(1.1 \mathrm{~Hz}, 0.24 \mathrm{~Hz})$. Thus, the method as it stands is incapable of detecting the presence of linear coupling between the oscillators by analysis of the test signal $x_{1 A}$. Nonetheless, we still suggest the use of bispectral analysis to investigate the presence of nonlinearity, but based on an adapted way of calculating the bispectrum.

In general, the bispectral method can be used to examine phase and frequency relationships at arbitrary time. It is thus well suited for detecting the presence of quadratic couplings and frequency modulation, since they both give rise to frequency components at the sum and difference of the interacting frequency components.

To be able to detect linear couplings using the bispectral method, as proposed, it is necessary to change the frequency relation. Study of coupled Poincaré oscillators demonstrate the presence of a component at frequency $2 k-l$ as a consequence of nonlinearity. This component was detected numerically, and is not necessarily characteristic of all nonlinear oscillators. By modifying the bispectral definition to

$$
B_{a}(k, l)=E\left[X(k) X(l) X^{*}(2 k-l)\right]
$$

the biphase turns into

$$
\phi_{a}(k, l)=\phi_{k}+\phi_{l}-\phi_{2 k-l}-\phi_{c},
$$

where index $a$ is introduced and will be used in what follows to indicate that the values are obtained using the adapted method. To obtain 0 radians in the case of phase coupling we have to correct the adapted biphase expression (9) by subtracting $\phi_{c}=2 \phi_{l}-\phi_{k}$. In the presence of a harmonically related frequency component and phase coupling, the biphase will then be 0 radians.

The adapted bispectrum $\left|B_{a}\right|$ for the signal $x_{1 A}$ exhibits several peaks, as shown in Fig. 3(a). It peaks where $f_{1}$ $=f_{2}$; a triple product $\left(f_{1}, f_{2}, f_{3}\right)$ of power at frequencies $f_{1}$ $=f_{2}=f$, and also $f_{3}=2 f_{1}-f_{2}=f$, raises a high peak at the bifrequency $(f, f)$. The self-coupling peak is physically meaningless, and it is therefore cut from the adapted bispectrum. It can be used for additional checking, since it strongly implies nonlinearity [6].

The peak of primary interest is at bifrequency $(1.1 \mathrm{~Hz}$, $0.24 \mathrm{~Hz}$ ). There is also a high peak positioned at bifrequency $(0.67 \mathrm{~Hz}, 0.24 \mathrm{~Hz})$ lying on the line where the third frequency in the triplet is equal to the frequency of the first oscillator and is therefore a consequence of the method. The small peaks present in the adapted bispectrum are the result of numerical rounding error and leakage effects due to the DFT calculation.

The peak $(1.1 \mathrm{~Hz}, 0.24 \mathrm{~Hz})$ indicates that oscillations at those pairs of frequencies are at least linearly frequency coupled. Frequency coupling alone is sufficient for a peak in the bispectrum to occur. Although the situation can in principle arise by coincidence, frequency and phase coupling together strongly imply the existence of nonlinearities. To be able to distinguish between different possible couplings, we calculate the adapted biphase Fig. 3(c).

During the first $400 \mathrm{~s}$ of test signal $x_{1 A}$, where no coupling is present, the adapted biphase changes continuously between 0 and $2 \pi$ radians. For the same time of observation it can be seen that the adapted biamplitude is 0, Fig. 3(d). During the second and third $400 \mathrm{~s}$ of the signal $x_{1 \mathrm{~A}}$, a constant adapted biphase can be observed indicating the pres- 

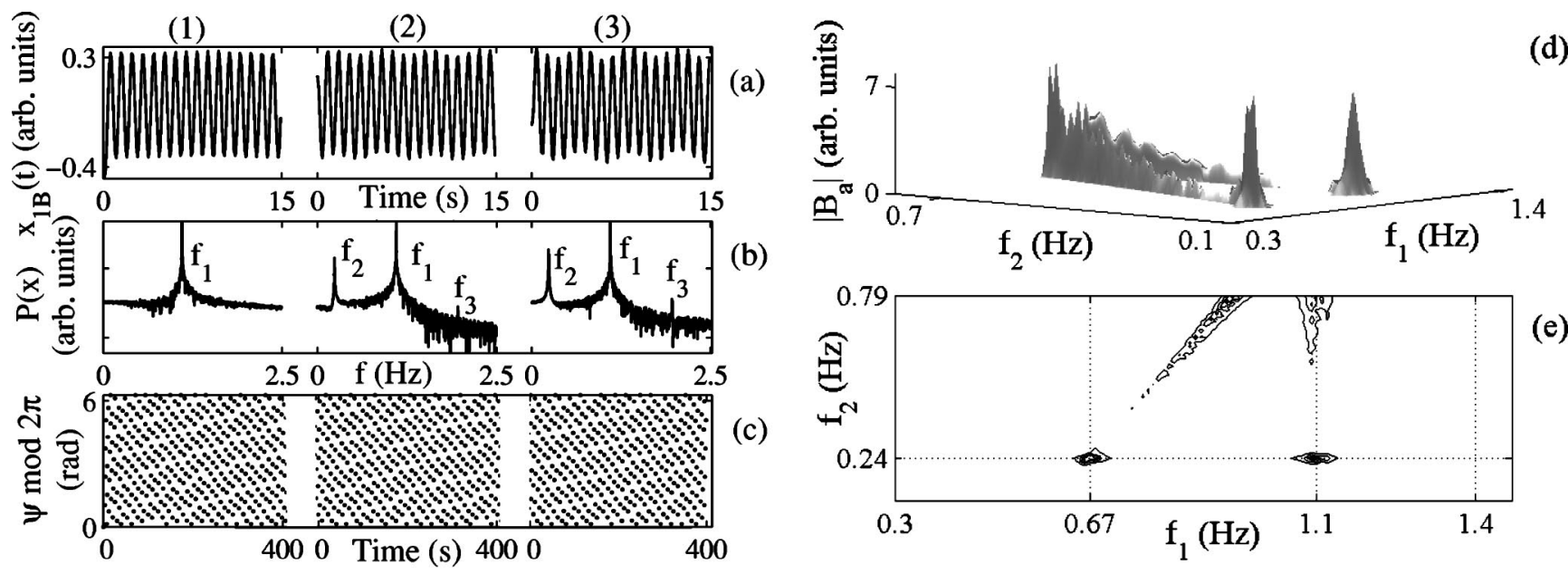

(d)
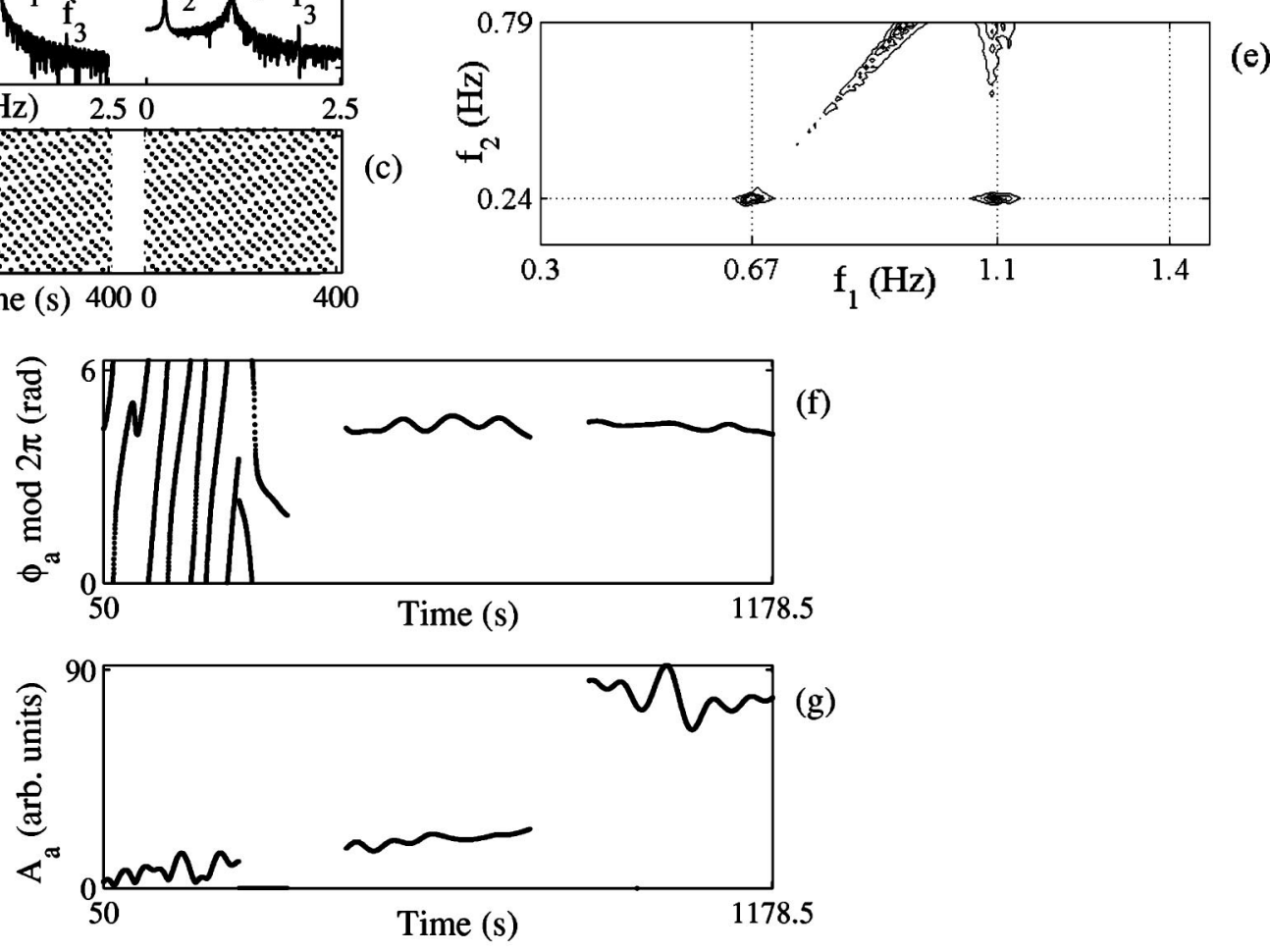

FIG. 4. Results in the presence of additive Gaussian noise. (a) Test signal $x_{1 B}$, variable $x_{1}$ of the first oscillator with characteristic frequency $f_{1}=1.1 \mathrm{~Hz}$. The characteristic frequency of the second oscillator is $f_{2}=0.24 \mathrm{~Hz}$. The oscillators are unidirectionally and linearly coupled with three different coupling strengths; $\eta_{2}=0.0$ (1), 0.1 (2), and 0.2 (3). Each coupling lasts for $400 \mathrm{~s}$ at a sampling frequency $f_{s}=10 \mathrm{~Hz}$. Only first $15 \mathrm{~s}$ are shown in each case. (b) Its power spectrum and (c) synchrogram. (d) Adapted bispectrum $\left|B_{a}\right|$ using $K$ $=33$ segments, $66 \%$ overlapping, and the Blackman window and (e) its contour view. The parts of the $\left|B_{a}\right|$ above $f_{2}>0.79 \mathrm{~Hz}$ and below $f_{1}<0.3 \mathrm{~Hz}$ are omitted because the triplets $(1.1 \mathrm{~Hz}, 1.1 \mathrm{~Hz}, 1.1 \mathrm{~Hz})$ and $(0.24 \mathrm{~Hz}, 0.24 \mathrm{~Hz}, 0.24 \mathrm{~Hz})$ produce a high peak that is physically meaningless. (f) Adapted biphase $\phi_{a}$ and (g) adapted biamplitude $A_{a}$ for bifrequency $(1.1 \mathrm{~Hz}, 0.24 \mathrm{~Hz})$, using a $0.3-\mathrm{s}$ time step and a 100 -s-long window for estimating the DFT using the Blackman window.

ence of linear coupling. The value of the adapted biamplitude is higher in the case of stronger coupling. The coupling constant $\eta_{2}$ can be obtained by normalization, and we are thus able to define the relative strengths of different couplings.

When the oscillators are coupled bidirectionally the frequency content of each of them changes and components $2 f_{1}$ and $2 f_{2}$ are generated. Both of these characteristic frequencies can be observed in the time series of each oscillator. Two combinatorial components are also present in their spectra, $2 f_{1}-f_{2}$ and $f_{1}-2 f_{2}$, assuming that $f_{1}>f_{2}$. In analyzing bidirectional coupling, the procedure described above can be extended and two combinatorial components should be analyzed in the same way.

Making use of the calculated instantaneous phases of both oscillatory components we also construct a synchrogram [Fig. 2(c)], as proposed by Schäfer et al. (see Ref. [1] and the references therein), and can immediately establish whether or not the coupling also results in synchronization.
The instantaneous phases can also be used to calculate the direction and strength of coupling, using the methods recently introduced by Schreiber, Rosenblum et al., and Paluš et al. [2].

\section{B. Linear couplings in the presence of noise}

We now test the method for the case where noise is added to the variable $x_{1}$ of the first oscillator:

$$
\begin{gathered}
\dot{x}_{1}=-x_{1} q_{1}-\omega_{1} y_{1}+g_{x_{1}}+\xi(t), \\
\dot{y}_{1}=-y_{1} q_{1}+\omega_{1} x_{1}+g_{y_{1}} .
\end{gathered}
$$

Here $\xi(t)$ is zero-mean white Gaussian noise, $\langle\xi(t)\rangle=0$, $\langle\xi(t), \xi(0)\rangle=D \delta(t)$, and $D=0.08$ is the noise intensity. In this way we obtain a test signal $x_{1 B}(t)$, Fig. 4(a). 

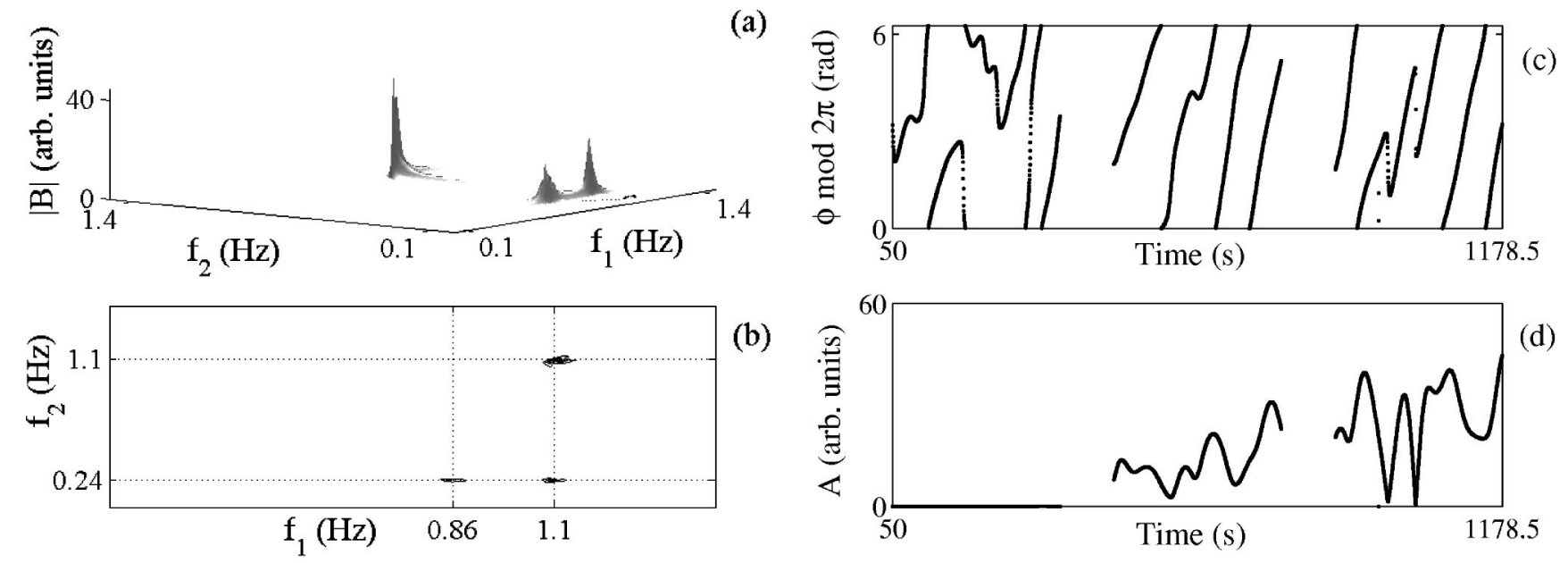

(b)
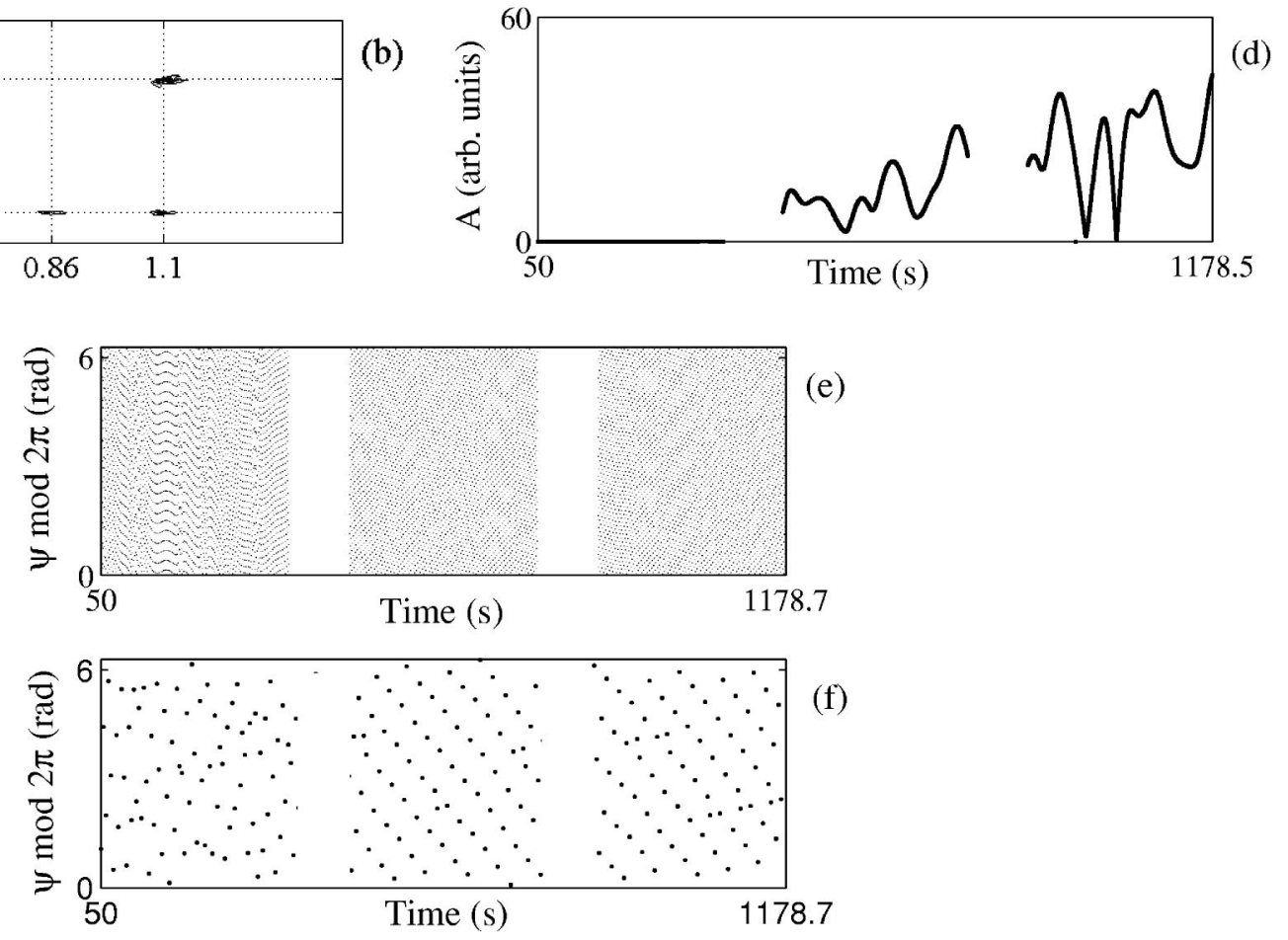

FIG. 5. Bispectrum $|B|$, calculated from the signal $x_{1 B}$ presented in Fig. 4(a), using $K=33$ segments, $66 \%$ overlapping, and the Blackman window to reduce leakage and (b) its contour view. (c) Biphase $\phi$ and (d) biamplitude $A$ for bifrequency $(1.1 \mathrm{~Hz}, 0.24 \mathrm{~Hz})$, using a 0.3 -s time step and a 100-s-long window for estimating the DFTs using a Blackman window. (e) Phase difference $\psi$ between $\phi_{1}$ of the characteristic frequency component $f_{1}$ of the first oscillator and $\phi_{2}$ of the characteristic frequency component $f_{2}$ of the second oscillator, for time step $1 / f_{s}$ and (f) at each period of lowest frequency $1 / f_{2}$ in the bifrequency pair $(1.1 \mathrm{~Hz}, 0.24 \mathrm{~Hz})$, using interpolation and 100 -s-long window for estimating DFTs using the Blackman window.

For nonzero coupling strength $\eta_{2}$, the component at frequency position $f_{3}$ can still be seen in the power spectrum, despite the noise, Fig. 4(b). The adapted biphase [Fig. 4(f)] can clearly distinguish between the presence and absence of coupling. When coupling is weaker, the adapted biamplitude [Fig. 3(g)] is lower and the adapted biphase is less constant.

The bispectrum for the signal $x_{1 B}$, shown in Fig. 5(a), differs from that in the case of zero noise, Fig. 2(d). Noise raises two additional peaks positioned at $(1.1 \mathrm{~Hz}, 0.24 \mathrm{~Hz})$ and $(0.86 \mathrm{~Hz}, 0.24 \mathrm{~Hz})$. The former could be the result of interaction; the latter is due to the method: the sum of the frequencies in this bifrequency pair gives the frequency of the first oscillator.

Close inspection of the $(0.24 \mathrm{~Hz}, 1.1 \mathrm{~Hz})$ peak by calculation of the biphase gives Fig. 5(c). When coupling is present, the characteristic frequency of the second oscillator appears in the power spectrum [Fig. 4(b)]. Two frequencies of high amplitude result in a small peak even if no harmonics are present at the sum and/or difference frequencies. The second peak is not of interest to us. It can easily be checked whether a phase coupling exists among the bifrequencies from the time evolution of the biphase.

In general, besides estimating bispectral values, one can also observe the time dependences of the phase and amplitude for each frequency component and their phase relationships. This applies particularly to frequencies that form a bifrequency giving a high peak in the bispectrum or adapted bispectrum. Synchrograms, Figs. 2(c) and 4(c), are obtained by first calculating the instantaneous phase of each oscillator and then their phase difference [1]. The phase difference in this case is between two fixed frequencies. We do not calculate their instantaneous frequencies, although it is possible to follow the frequency variation by calculating the phase difference at neighboring bifrequencies around the observed one and showing them simultaneously on the same plot. Examples of the phase difference $\psi=\phi_{1}-\phi_{2}$ between the phases of the first $\phi_{1}$ and the second $\phi_{2}$ interacting oscillators are shown in Figs. 5(e) and 5(f). 

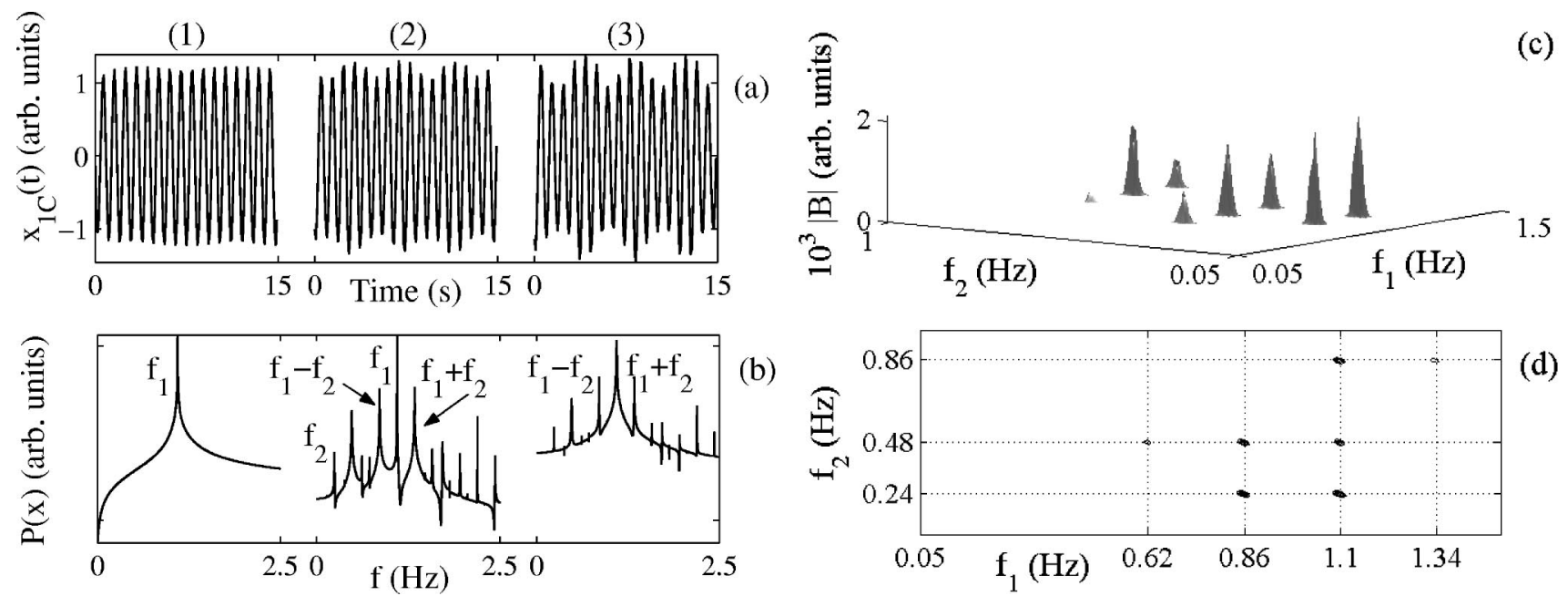

(b)

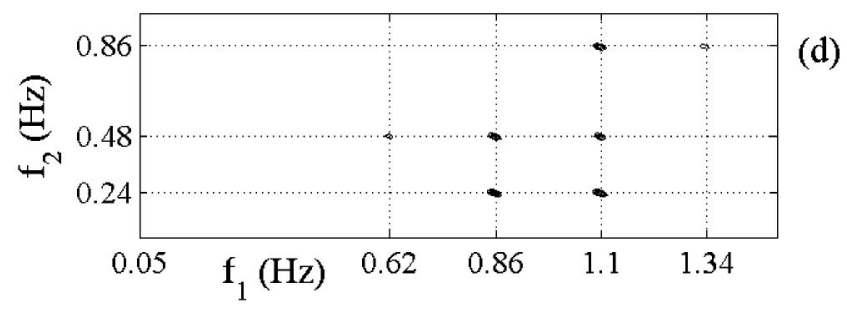

FIG. 6. Results for quadratic coupling in the absence of noise. (a) The test signal $x_{1 C}$, variable $x_{1}$ of the first oscillator with characteristic frequency $f_{1}=1.1 \mathrm{~Hz}$. The characteristic frequency of the second oscillator is $f_{2}=0.24 \mathrm{~Hz}$. Oscillators are unidirectionally and quadratically coupled with three different coupling strengths: $\eta_{2}=0.0$ (1), 0.05 (2), and 0.1 (3). Each coupling lasts for $400 \mathrm{~s}$ at sampling frequency $f_{s}$ $=10 \mathrm{~Hz}$. Only the first $15 \mathrm{~s}$ are shown in each case. (b) The power spectrum. (c) The bispectrum $|B|$, using $K=33$ segments, $66 \%$ overlapping, and the Blackman window to reduce leakage and (d) its contour view. The part of the bispectrum above $f_{2}>1.0 \mathrm{~Hz}$ is cut, because triplet $(1.1 \mathrm{~Hz}, 1.1 \mathrm{~Hz}, 1.1 \mathrm{~Hz})$ produces a high peak that is not physically significant.

\section{Quadratic couplings}

We now assume that two Poincaré oscillators can interact with each other nonlinearly. A quadratic nonlinear interaction generates higher harmonic components in addition to the characteristic frequencies [5]. In order to study an example where the first $f_{1}=1.1 \mathrm{~Hz}$ and second $f_{2}=0.24 \mathrm{~Hz}$ oscillators are quadratically coupled, we change the coupling terms in model (6) to quadratic ones

$$
g_{x_{1}}=\eta_{2}\left(x_{1}-x_{2}\right)^{2}, \quad g_{y_{1}}=\eta_{2}\left(y_{1}-y_{2}\right)^{2} .
$$

Clearly, the test signal $x_{1 C}$ presented in Fig. 6(a) for three different coupling strengths [no coupling $\eta_{2}=0$ (1) and weak couplings $\eta_{2}=0.05(2), \eta_{2}=0.1$ (3)] has a richer harmonic structure. In addition to the characteristic frequencies, it contains components with frequencies $2 f_{1}, 2 f_{2}, f_{1}+f_{2}$, and $f_{1}-f_{2}$ [Fig. 6(b)]. Equation (11) also indicates that, as well as having a particular harmonic structure, the components of the signal $x_{1 C}$ also have related phases, $2 \phi_{1}, 2 \phi_{2}, \phi_{1}+\phi_{2}$, and $\phi_{1}-\phi_{2}$.

We expect several peaks [24] to arise in the bispectrum. The peak of principal interest is at bifrequency $(1.1 \mathrm{~Hz}, 0.24$ $\mathrm{Hz})$. As before, the self-coupling peaks are at $(1.1 \mathrm{~Hz}, 1.1 \mathrm{~Hz})$ and $(0.24 \mathrm{~Hz}, 0.24 \mathrm{~Hz})$ are of no interest, so they are cut from the bispectrum. Additional peaks appear at the bifrequencies $(0.86 \mathrm{~Hz}, 0.24 \mathrm{~Hz}),(0.62 \mathrm{~Hz}, 0.48 \mathrm{~Hz}),(0.86 \mathrm{~Hz}, 0.48 \mathrm{~Hz})$, $(1.1 \mathrm{~Hz}, 0.48 \mathrm{~Hz}),(1.1 \mathrm{~Hz}, 0.86 \mathrm{~Hz})$, and $(1.34 \mathrm{~Hz}, 0.86 \mathrm{~Hz})$. The triplet of harmonically related frequency components $\left(f_{1}, f_{2}, f_{3}\right)$ would peak in the bispectrum when the power for all these frequencies differs from zero. The components 0.48 $\mathrm{Hz}, 0.86 \mathrm{~Hz}, 1.34 \mathrm{~Hz}$, and $2.2 \mathrm{~Hz}$ resulting from quadratic couplings form such triplets that peak in the bispectrum: $(0.86 \mathrm{~Hz}, 0.24 \mathrm{~Hz}, 1.1 \mathrm{~Hz}),(0.86 \mathrm{~Hz}, 0.48 \mathrm{~Hz}, 1.34 \mathrm{~Hz})$, and (1.34 Hz,0.86 Hz,2.2 Hz). Besides these, there are also other peaks, e.g., that at the bifrequency $(0.62 \mathrm{~Hz}, 0.48 \mathrm{~Hz})$ arising from the triplet $(0.62 \mathrm{~Hz}, 0.48 \mathrm{~Hz}, 1.1 \mathrm{~Hz})$; the sum-difference combination of such frequencies always give the characteristic frequency, or one that results from quadratic coupling. The existence of such peaks has no other meaning than as a strong indicator of second-order nonlinearity. Consequently, the biphase for all peaks due to possible nonlinear mechanisms in the bispectrum must have the same value, and same behavior, as shown, e.g., in Figs. 7(a) and 7(c). The biphase is constant in the presence of quadratic coupling. From the biamplitude, the coupling constant can be determined by normalization.

In the power spectrum there is a component at frequency $2 f_{1}-f_{2}$, even although linear coupling is absent. It arises from nonlinearity in the Poincare oscillator. The adapted bispectrum for the signal $x_{1 C}$ shows a peak at bifrequency $(1.1 \mathrm{~Hz}, 0.24 \mathrm{~Hz})$, but the adapted biphase varies continuously: we may therefore exclude the possibility of linear coupling being present.

\section{Quadratic couplings in the presence of noise}

As in the case of linear coupling (Sec. II B) we add a noise term to the quadratic coupling $g_{x_{1}}$ and obtain the test signal $x_{1 D}$, presented in Fig. 8(a).

Using the bispectral and adapted bispectral methods, we find that we obtain results very similar to those in the absence of noise. The method is evidently noise robust. The results for nonzero coupling are quite different from those where coupling is absent, Fig. 8(e).

\section{E. Frequency modulation in the presence of noise}

We are also interested of being able to detect parametric frequency modulation and to distinguish it from quadratic coupling. Parametric modulation produces frequency components at the sum and difference of the characteristic fre- 

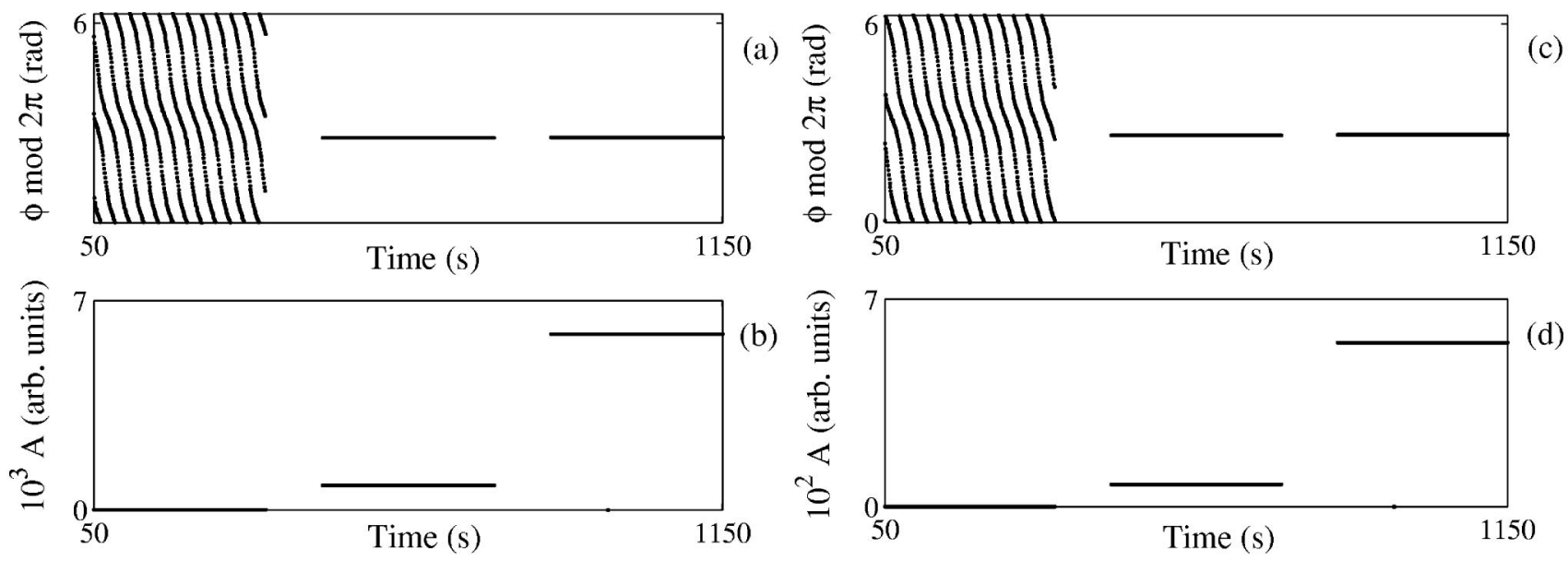

FIG. 7. (a) The biphase $\phi$ and (b) biamplitude $A$ for the test signal $x_{1 C}$ for bifrequency $(1.1 \mathrm{~Hz}, 0.24 \mathrm{~Hz})$, using $0.3-\mathrm{s}$ time step and 100 -s-long window for estimating DFT using the Blackman window. (c) Biphase and (d) biamplitude for the bifrequency $(0.86 \mathrm{~Hz}, 0.24 \mathrm{~Hz})$, with a 0.3-s time step and a 100-s-long window for estimating DFT using the Blackman window.

quency and the modulation frequency, i.e., the same two frequency components that can also result from quadratic coupling. Let us now consider an example where the first oscillator $f_{1}=1.1 \mathrm{~Hz}$ is frequency modulated by the second one $f_{2}=0.24 \mathrm{~Hz}$. For this purpose the equations of the first oscillator become

$$
\begin{gathered}
\dot{x}_{1}=-x_{1} q_{1}-y_{1}\left(\omega_{1}+\eta_{m} x_{2}\right)+\xi(t), \\
\dot{y}_{1}=-y_{1} q_{1}+x_{1}\left(\omega_{1}+\eta_{m} y_{2}\right) .
\end{gathered}
$$

The model parameters $\alpha_{1,2}, a_{1,2}$ and the noise intensity $D$ are chosen to be the same as in the previous examples.

We thus obtain a test signal $x_{1 E}$. It is the time evolution of the variable $x_{1}$ of the first oscillator, presented in Fig. 9(a) with the corresponding power spectrum 9(b) for three different parametric frequency modulation strengths: no modulation $\eta_{m}=0$; and modulation $\eta_{m}=0.1,0.2$. The bispectrum of the test signal $x_{1 E}$, Fig. 9(c), exhibits several high peaks. The highest are at bifrequencies $(1.1 \mathrm{~Hz}, 0.86 \mathrm{~Hz}),(0.86 \mathrm{~Hz}$, $0.24 \mathrm{~Hz})$, and $(1.1 \mathrm{~Hz}, 0.24 \mathrm{~Hz})$, in addition to the $(1.1 \mathrm{~Hz}$, $1.1 \mathrm{~Hz}$ ) peak. They also appear in the case of quadratic coupling. In general, however, the other peaks that appear for quadratic coupling are absent. The reason is that although the component of the second oscillator $f_{2}$ (one component of the triplet) is not present in the power spectrum, its value is not not exactly zero.

Observing the biphase, no epochs of constant biphase can be observed, although for strong frequency modulation the biphase is less variable. In the power spectrum, Fig. 9(b), no component rises above the noise level at frequency $f_{2}$, of the bifrequency pair, where the bispectrum peaks. This is an indication that there is parametric coupling between the oscillators, as there is a high value of biamplitude. The biphase changes runs between 0 and $2 \pi$, and is modulated in the absence of noise. There are also no rapid $2 \pi$ phase slips of the kind that are normal if no modulation is present. In the absence of couplings and modulation, but in the presence of noise, there would be no such peaks in the power spectrum or bispectrum.

\section{SUMMARY AND CONCLUSIONS}

We have extended the bispectral method to encompass time dependence and have demonstrated the potential of the extended technique to determine the type of coupling among interacting nonlinear oscillators. Time-phase couplings can be observed by calculating the bispectrum and adapted bispectrum and by obtaining the time-dependent biphase and biamplitude. The method has the advantage that it allows an arbitrary number of interacting oscillatory processes to be studied.

Recently introduced methods for synchronization analysis among chaotic and noisy oscillations (see Ref. [1] and references therein) have stimulated applications to a variety of different systems. Methods for quantifying the strength and identifying the direction of couplings, based on nonlinear dynamic or information theory approaches, have recently been proposed [2]. Here we have addressed the question of the type of coupling that may result in synchronization, and we have proposed a method for its analysis. It is applicable to both univariate data (a single signal from the coupled system) or multivariate data (a separate signal from each oscillator).

Millingen et al. [15] have analyzed multivariate data using a combined wavelet and bispectral method, and have discussed its application in the field of chaos analysis. Here we have concentrated on univariate data and illustrated the potential of the time-phase bispectral method for the detection of higher-order couplings in the presence of noise. The possibility of using univariate data is of particular importance when dealing with real signals, as in practice we often cannot observe and measure the separate subsystems directly, but only their combination, which is intrinsically difficult. Most of the methods proposed so far for synchronization analysis and detection of the direction of couplings are based 

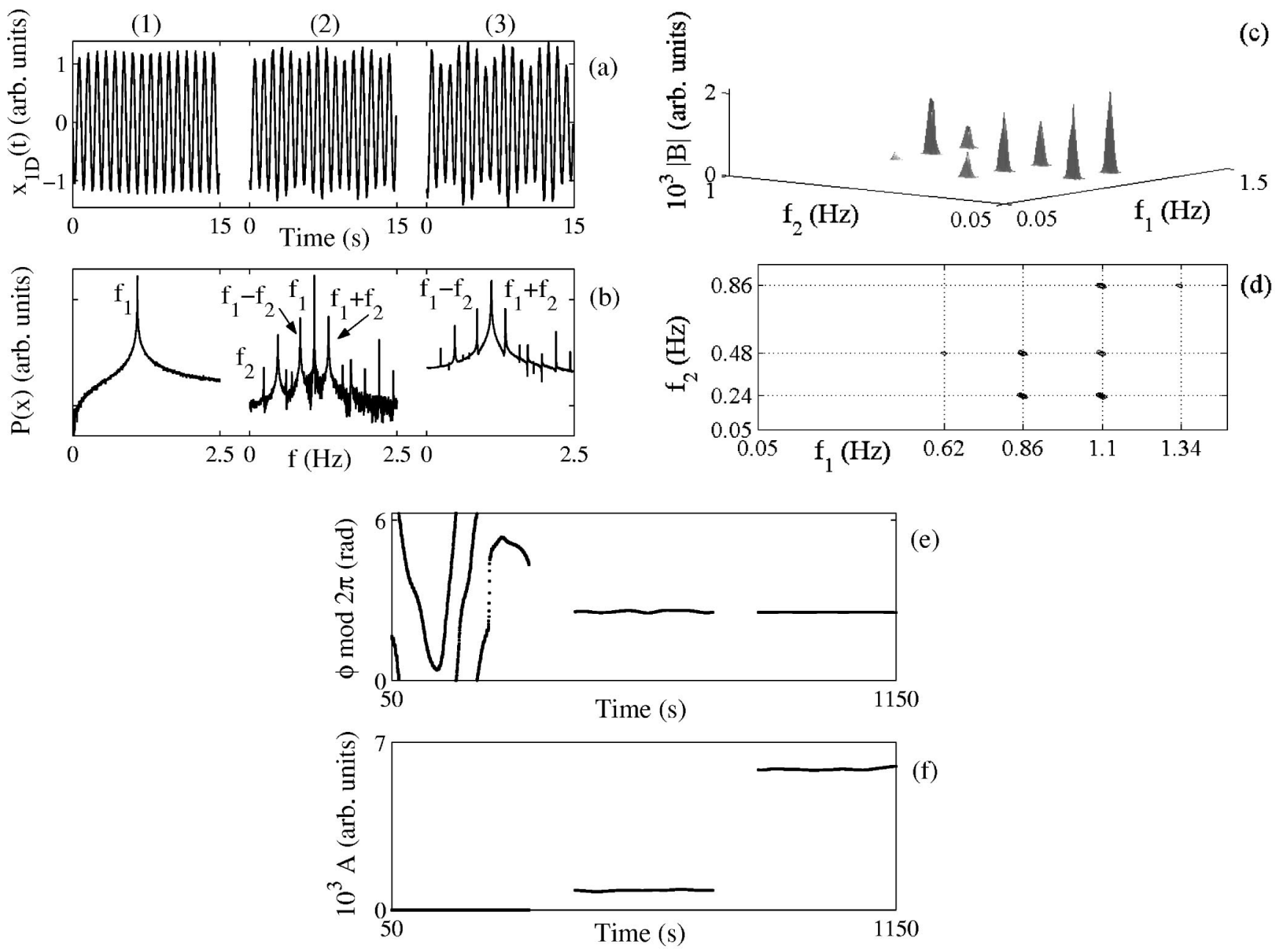

FIG. 8. Results for quadratic couplings in the presence of additive Gaussian noise. (a) The test signal $x_{1 D}$, variable $x_{1}$ of the first oscillator with characteristic frequency $f_{1}=1.1 \mathrm{~Hz}$. The characteristic frequency of the second oscillator is $f_{2}=0.24 \mathrm{~Hz}$. The oscillators are unidirectionally and quadratically coupled with three different coupling strengths: $\eta_{2}=0.0$ (1), 0.05 (2), and 0.1 (3). Each coupling lasts for $400 \mathrm{~s}$ at a sampling frequency $f_{s}=10 \mathrm{~Hz}$. Only the first $15 \mathrm{~s}$ are shown in each case. (b) The power spectrum. (c) The bispectrum $|B|$ calculated with $K=33$ segments, $66 \%$ overlapping, and using the Blackman window to reduce leakage and (d) its contour view. The part of the bispectrum above $f_{2}>1.0 \mathrm{~Hz}$ is cut, because the triplet $(1.1 \mathrm{~Hz}, 1.1 \mathrm{~Hz}, 1.1 \mathrm{~Hz}$ ) produce a high peak that is physically meaningless. (e) The biphase $\phi$ and (f) biamplitude $A$ for bifrequency $(1.1 \mathrm{~Hz}, 0.24 \mathrm{~Hz})$, with a 0.3 -s time step and a 100-s-long window for estimating DFTs using the Blackman window.

on bivariate or multivariate data $[1,2]$. In conjunction with frequency or time-frequency filtering [27] or mode decomposition [28] to obtain two or more "separate" signals, these methods can be used for univariate data as well. Synchronization can also be detected in univariate data through an analysis of angles and radii [29] in return time maps [30].

The time-phase bispectral method proposed in this paper is not only applicable to the synchronization analysis of univariate data but also, at the same time, allows one to determine the nature of the couplings among the interacting nonlinear oscillators. Its benefits include (1) the possibility of observing the whole frequency domain simultaneously; (2) detecting that two or more subsystems are interacting with each other; (3) quantification of the strength of the interaction; and (4) determination of whether the coupling is additive linear or quadratic, or parametric in one of the frequen- cies. We have shown the method to be suitable for the analysis of noisy signals.

Although we have shown that the technique works effectively on a well-characterized simple model, there will be some difficulties to be faced and overcome in applying it to real problems, e.g., to data from the cardiovascular system. Understanding the content of the bispectrum and identification of the peaks of interest are not always straightforward. To appreciate which peaks are those to focus on, one has to be aware of the basic properties of the system and its fundamental frequencies. Distinguishing a quadratic interaction from parametric frequency modulation may be easy when the coupling (modulation) is relatively strong, but becomes more difficult in the case of relatively weak coupling (modulation). In the latter case, observing each phase in the triplet separately can be helpful. Also it is not always an easy task 

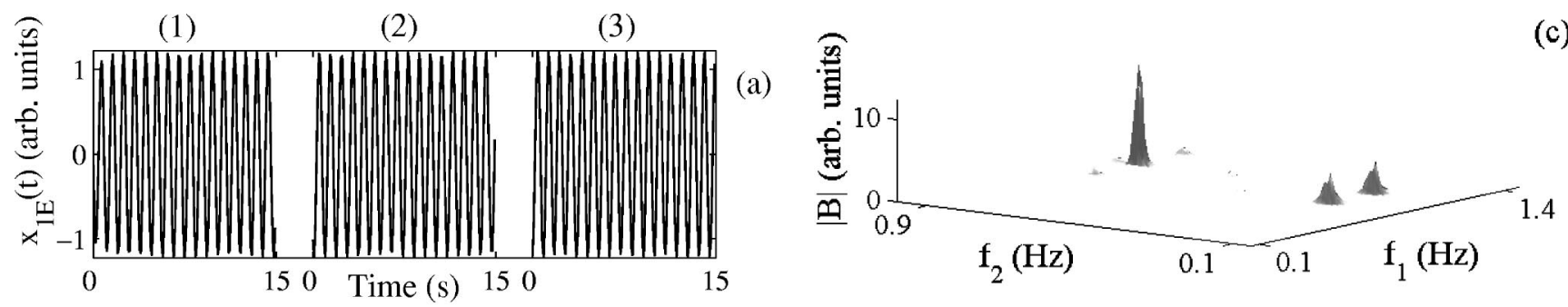

(c)
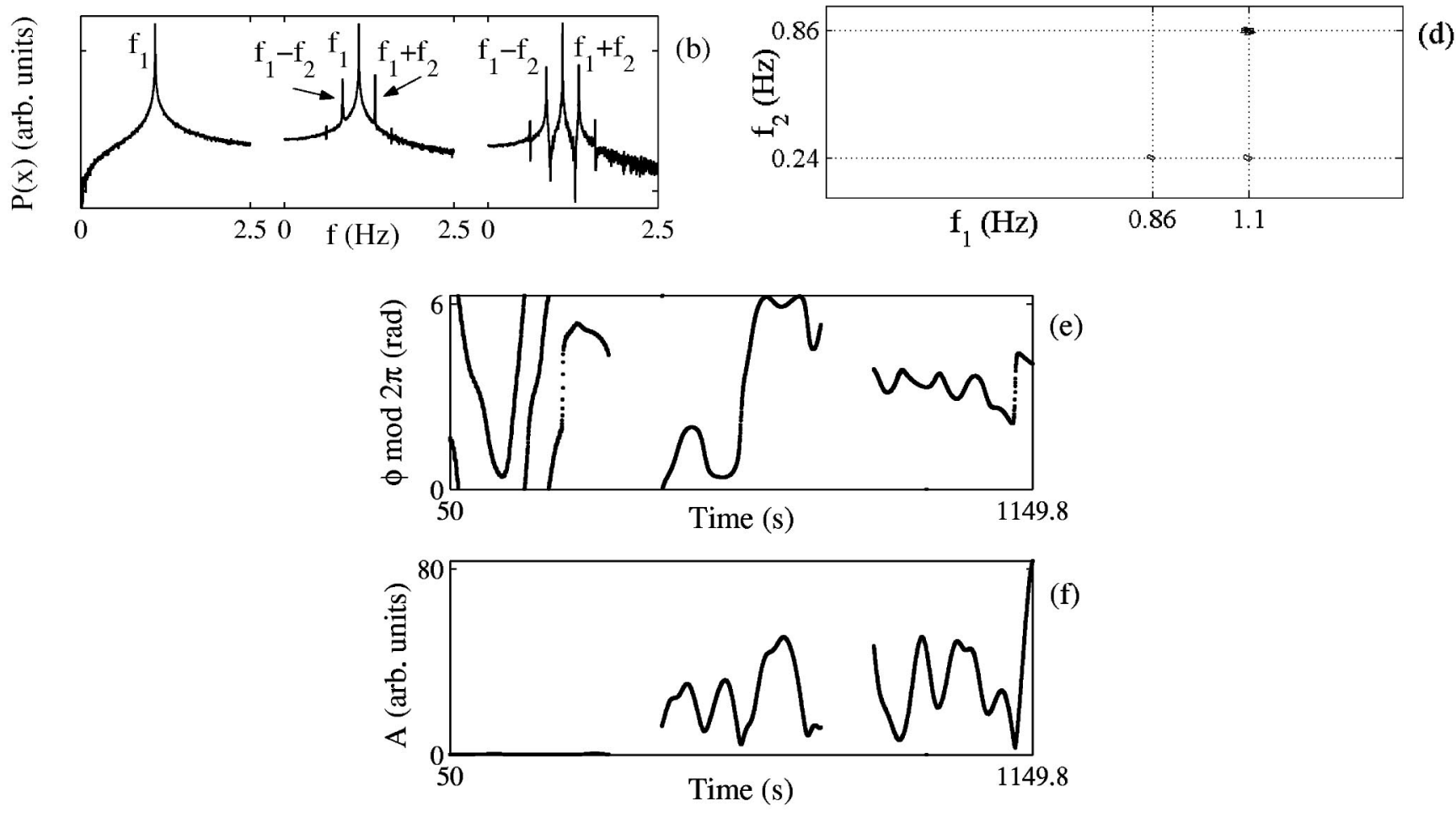

FIG. 9. Results for parametric frequency modulation in the presence of additive Gaussian noise. (a) The test signal $x_{1 E}$, of variable $x_{1}$ of the first oscillator with characteristic frequency $f_{1}=1.1 \mathrm{~Hz}$ frequency modulated by the second oscillator $f_{2}=0.24 \mathrm{~Hz}$ with three different frequency modulation strengths: $\eta_{m}=0.0$ (1), 0.1 (2), and 0.2 (3). Each frequency modulation lasts for $400 \mathrm{~s}$, at sampling frequency $f_{s}$ $=10 \mathrm{~Hz}$. Only the first $15 \mathrm{~s}$ are shown in each case. (b) The power spectrum. (c) The bispectrum $|B|$ calculated with $K=33 \mathrm{segments}, 66 \%$ overlapping, and using the Blackman window to reduce leakage and (d) its contour view. The part of the bispectrum above $f_{2}>1.0 \mathrm{~Hz}$ is cut, because the triplet $(1.1 \mathrm{~Hz}, 1.1 \mathrm{~Hz}, 2.2 \mathrm{~Hz})$ produces a high peak that is physically meaningless. (e) The biphase $\phi$ and (f) biamplitude $A$ for bifrequency $(1.1 \mathrm{~Hz}, 0.24 \mathrm{~Hz}$ ), with a 0.3-s time step and a 100-s-long window for estimating the DFTs using the Blackman window.

to distinguish between quadratic interaction and parametric frequency modulation in the cases when both of them occur simultaneously. Further, where the possible basic frequencies are relatively close, it will be hard to detect them separately. This could cause particular problems in the detection of quadratic phase couplings where frequency pairs are close together. Although it is possible in principle to study an arbitrary number of interacting oscillators, it is advisable in practice to study them in pairs: a knowledge of the basic frequency of each is necessary.

The time-dependent biphase-biamplitude estimate was estimated with a short-time Fourier transform (STFT), using a window of constant length. The optimal window length depends, however, on the frequency being studied. The effective length of the window used for each frequency can be varied by applying the wavelet transform, or the selective Fourier transform. For demonstration purposes above, the natural frequencies of the oscillators were chosen to lie within a relatively narrow frequency interval. A STFT was therefore sufficient for good time and phase (frequency) localization. With a broader frequency content, however, the wavelet transform or selective Fourier transform will need to be applied.

Higher-order spectral methods can be used to study arbitrary interactions among coupled oscillators: of quadratic, cubic, or even higher order. In this paper we have concentrated on the lowest one, using the third-order spectrum or bispectrum. For higher orders the volume of the calculations rises substantially, and the method becomes numerically increasingly demanding. At the same time, graphical presentation and interpretation of the results become increasingly difficult.

\section{ACKNOWLEDGMENTS}

We gratefully acknowledge valuable comments and discussions with Andriy Bandrivskyy, Justin Fackrell, Mounir 
Ghogho, Fluvio Gini, Georgios B. Giannakis, Peter Husar, Dmitri G. Luchinsky, and Anathram Swami. The study was supported by the Slovenian Ministry of Education, Science and Sport, by INTAS, and by the Engineering and Physical Sciences Research Council (U.K.).

\section{APPENDIX: VARIANCE OF THE BISPECTRUM ESTIMATE}

In order to interpret bispectral values from a finite length time series, the statistics of bispectrum estimates must be known. To achieve statistical stability, the time series is divided into $K$ segments for averaging [25]. When there is a large number of segments, the estimate gains statistical stability at the expense of power spectral and bispectral resolution. For a real signal, with a finite number of points, the compromise between bispectral resolution and statistical stability may be expected at $K$ around 30. Estimates are subject to statistical error, such as bias and variance. An estimate must be consistent, that is the statistical error must approach zero in the mean-square sense as the number of realizations becomes infinite. Here we neglect the effects of finite time series length, we assume that they are sufficiently long. Let us consider the bias and the variance of the bispectrum estimate $\hat{B}(k, l)$. The expected value of $\hat{B}(k, l)$ will be

$$
\begin{aligned}
E[\hat{B}(k, l)] & =\frac{1}{K} \sum_{i=1}^{K} E\left[X_{i}(k) X_{i}(l) X_{i}^{*}(l, k)\right] \\
& =E\left[X(k) X(l) X^{*}(l, k)\right]=B(k, l),
\end{aligned}
$$

as $K$ becomes infinite, $X_{i}$ is the DFT of the $i$ th segment. Thus, $\hat{B}(k, l)$ can be taken as an unbiased estimate [29]. Its variance will be

$$
\begin{aligned}
\operatorname{var}(\hat{B}) & =E\left[\hat{B} \hat{B}^{*}\right]-E[\hat{B}] E\left[\hat{B}^{*}\right] \\
& =\frac{1}{K}\left\{E\left[|X(k)|^{2}|X(l)|^{2}|X(k+l)|^{2}\right]-E|B(k, l)|^{2}\right\} .
\end{aligned}
$$

Note that the variance is inversely proportional to $K$. From a mathematical statistics point of view, it is a nontrivial task to compute the quantity in the bracket in terms of low order spectra, but one may write a good approximation [26],

$$
E\left[|X(k)|^{2}|X(l)|^{2}|X(k+l)|^{2}\right]=P(k) P(l) P(k+l),
$$

in which case the variance will be

$$
\begin{aligned}
\operatorname{var}(\hat{B}) & =E\left[|\hat{B}(k, l)|^{2}\right]-E[\hat{B}(k, l)]^{2} \\
& \approx \frac{1}{K} P(k) P(l) P(k+l)\left[1-b^{2}(k, l)\right] .
\end{aligned}
$$

Note that it is a consistent estimate in the sense that the variance approaches zero as $K$ becomes infinite. The variance is proportional to the product of the powers $[P(k)$ $\left.=E\left[X(k) X^{*}(k)\right]\right]$ at the frequencies $k, l$, and $k+l$. Consequently, a larger statistical variability is introduced in estimating larger values in the bispectrum. Finally, the variance is proportional to $\left[1-b^{2}(k, l)\right]$, where the bicoherence $b^{2}$ is a normalized bispectrum, $\quad b^{2}(k, l)=E[\hat{B}(k, l)]^{2} /$ $[P(k) P(l) P(k+l)]$. That is, when the oscillations at $k, l$, and $k+l$ are nonlinearly coupled $\left(b^{2} \approx 1\right)$, the variance approaches zero, and when the components are statistically independent $\left(b^{2} \approx 0\right)$, the variance is proportional to the power at each spectral component [26].

Brillinger and Rosenblatt [3] have investigated the asymptotic mean and variance of Fourier-type estimates of high-order spectra and proved that under certain assumptions the $k$ th order spectral estimate is asymptotically unbiased and Gaussianly distributed and that estimates of different order are asymptotically independent. The variances of the real and imaginary parts of the bispectrum are asymptotically (i.e., for large $K$ ) Gaussian and are equal, $\operatorname{var}\{\operatorname{Re}[\hat{B}(k, l)]\}$ $\cong \operatorname{var}\{\operatorname{Im}[\hat{B}(k, l)]\}$. For a perfect phase-coupled triplet, the variances of the real and imaginary parts are equal to zero. In the case of no coupling, there is an identical contribution to the variances from the real and imaginary parts of the estimate of the bispectrum.

The total variance is a sum of individual $(i=1, \ldots, K)$ contributions, because different triplets are mutually statistically uncorrelated in the absence of phase coupling. Partial coupling can be expected to result in a combination of perfectly phase-coupled oscillations and oscillations with randomly changing phases.
[1] A.S. Pikovsky, M.G. Rosenblum, and J. Kurths, Synchronization; A Universal Concept in Nonlinear Sciences (Cambridge University Press, Cambridge, 2001).

[2] T. Schreiber, Phys. Rev. Lett. 85, 461 (2000); M.G. Rosenblum and A.S. Pikovsky, Phys. Rev. E 64, 045202 (2001); M.G. Rosenblum, L. Cimponeriu, A. Bezerianos, A. Patzak, and R. Mrowka, ibid. 65, 041909 (2002); M. Paluš, V. Komárek, Z. Hrnčĩr, and K. Štěbrová, ibid. 63, 046211 (2001).

[3] D.R. Brillinger and M. Rosenblatt, Spectral Analysis of Time Series (Wiley, New York, 1967).
[4] A. Swami, G.B. Giannakis, and G. Zhou, Signal Process. 60, 65 (1997).

[5] C.L. Nikias and A.P. Petropulu, Higher-Order Spectra Anlysis: A Nonlinear Signal Processing Framework (Prentice-Hall, Englewood Cliffs, 1993).

[6] G. Zhou and G.B. Giannakis, IEEE Trans. Signal Process. 43, 1173 (1995).

[7] J.W.A. Fackrell, Ph.D. thesis, University of Edinburgh, 1996 (unpublished).

[8] Y.C. Kim, J.M. Beall, E.J. Powers, and R.W. Miksad, Phys. 
Fluids 23, 258 (1980); M.R. Raghuveer, IEEE Trans. Autom. Control 35, 48 (1990); H. Parthasarathy, S. Prasad, and S.D. Joshi, IEEE Trans. Signal Process. 43, 2346 (1995).

[9] M.B. Priestley and M.M. Gabr, Multivariate Analysis: Future Directions (North-Holland, Amsterdam, 1993).

[10] J.R. Fonollosa and C.L. Nikias, IEEE Trans. Signal Process. 41, 245 (1993); B. Boashash and P.J. O'Shera, ibid. 42, 216 (1994).

[11] T.S. Rao and K.C. Indukumar, J. Franklin Inst. 33, 425 (1996).

[12] R.J. Perry and M.G. Amin, IEEE Trans. Signal Process. 43, 1017 (1995).

[13] A.V. Dandawaté and G.B. Giannakis, IEEE Trans. Inf. Theory 41, 216 (1995).

[14] B. Schack et al., Clin. Neurophysiol. 112, 1388 (2001).

[15] B.Ph. van Milligen, C. Hidalgo, and E. Sánchez, Phys. Rev. Lett. 74, 395 (1995); B.Ph. van Milligen et al., Phys. Plasmas 2, 3017 (1995).

[16] J. Jamšek, M.Sc. thesis, University of Ljubljana, 2000.

[17] A. Stefanovska and M. Bračič, Contemp. Phys. 40, 31 (1999).

[18] J.M. Mendel, Proc. IEEE 79, 278 (1991).

[19] A.K. Nadi, IEE Proc. F, Commun. Radar Signal Process. 140, 380 (1993).

[20] A.K. Nadi, Higher-Order Statistics in Signal Processing (Cambridge University Press, Cambridge, 1998).
[21] C.L. Nikias and J.M. Mendel, IEEE Signal Process. Mag. 7, 10 (1993).

[22] L.A. Pflug, G.E. Ioup, and J.W. Ioup, J. Acoust. Soc. Am. 94, 2159 (1993); 95, 2762 (1994).

[23] M.J. Hinch, IEEE Trans. Acoust., Speech, Signal Process. 38, 1277 (1990); I. Sharfer and H. Messer, IEEE Trans. Signal Process. 41, 296 (1993); M.J. Hinch, ibid. 43, 2130 (1995).

[24] Three and not four, because the triplet $\left(f_{1}, f_{2}, f_{1}+f_{2}\right)$ has the same peak in the bispectrum as the triplet $\left(f_{1}, f_{2}, f_{1}-f_{2}\right)$.

[25] The phases are random variables over $[0.2 \pi)$. The phases of different segments are independent of each other.

[26] Y.C. Kin and E.J. Powers, IEEE Trans. Plasma Sci. PS-7, 120 (1979); V. Chandran, Ph.D. thesis, Washington State University, 1990; V. Chandran and S.L. Elgar, IEEE Trans. Signal Process. 19, 2640 (1991).

[27] P. Tass, M.G. Rosenblum, J. Weule, J. Kurths, A. Pikovsky, J. Volkmann, A. Schnitzler, and H.-J. Freund, Phys. Rev. Lett. 81, 3291 (1998); A. Stefanovska and M. Hožič, Prog. Theor. Phys. Suppl. 139, 270 (2000).

[28] N.E. Huang, Z. Shen, S.R. Long, M.C. Wu, H.H. Shih, Q. Zheng, N. Yen, C.C. Tung, and H.H. Liu, Proc. R. Soc. London, Ser. A 454, 903 (1998).

[29] N.B. Janson, A.G. Balanov, V.S. Anishchenko, and P.V.E. McClintock, Phys. Rev. E 65, 036211 (2002).

[30] K. Suder, F.R. Drepper, M. Schiek, and H.H. Abel, Am. J. Physiol. Heart Circ. Physiol. 275, H1092 (1998). 